\title{
Relato de Experiência sobre o uso da Computação Desplugada associada a uma Teoria de Aprendizagem Colaborativa
}

\author{
Rachel C. D. Reis ${ }^{1,2}$, Kamila T. Lyra ${ }^{1}$, Clausius D. G. Reis ${ }^{1,2}$, Seiji Isotani ${ }^{1}$ \\ ${ }^{1}$ Instituto de Ciências Matemáticas e de Computação - Universidade de São Paulo, \\ Avenida Trabalhador São Carlense, 400 - Centro - 13566-590 - São Carlos, SP \\ ${ }^{2}$ Universidade Federal de Viçosa, Campus Rio Paranaíba \\ rachel.reis@ufv.br, kalyra 03@usp.br, clausius@ufv.br, \\ sisotani@ìcmc.usp.br
}

\begin{abstract}
The use of Computer Science Unplugged to support computing teaching has been evaluated in different learning scenarios. Despite of important contributions, we observed the lack of studies that analyze the use of this technique in collaborative scenarios based on learning theories. Therefore, this paper aims at investigating the use of the Computer Science Unplugged technique in learning groups based on Peer Tutoring theory. A quantitative experimental study, carried out with 162 elementary students, showed a significant improvement in students' learning. In addition, we presented the most common mistakes made by students in such scenario.
\end{abstract}

Resumo. $O$ uso da Computação Desplugada para apoiar o ensino de Computação tem sido avaliado em diferentes cenários de aprendizagem. Apesar das importantes contribuições, observou-se a carência de estudos que analisem o uso dessa técnica em cenários colaborativos fundamentados por teorias de aprendizagem. Logo, este artigo tem como objetivo investigar o uso da técnica de Computação Desplugada em grupos de aprendizagem fundamentados pela teoria Peer Tutoring. Um estudo experimental quantitativo, realizado com 162 alunos do ensino fundamental II, mostrou uma melhora significativa no aprendizado dos alunos. Além disso, foram mostrados os principais erros cometidos pelos alunos neste cenário.

\section{Introdução}

A técnica de Computação Desplugada foi criada com o intuito de possibilitar que indivíduos sem acesso ao computador tivessem a oportunidade de entender o seu funcionamento e seus fundamentos [Bell et al. 2009]. Por ser de fácil compreensão, diversos pesquisadores na literatura têm utilizado essa técnica como uma forma de dinamizar o aprendizado dos conceitos de computação [Vieira et al. 2013, Barbosa et al. 2015], bem como uma abordagem de ensino para a explicação de conteúdos mais específicos da área de Computação [Costa et al. 2017, Martinhago et al. 2014].

Com base nos benefícios da técnica de Computação Desplugada para a aprendizagem e satisfação dos alunos, diversos trabalhos na literatura têm investigado seu uso com públicos de diferentes níveis de escolaridade e em diferentes cenários de 
VII Congresso Brasileiro de Informática na Educação (CBIE 2018)

Anais do XXIV Workshop de Informática na Escola (WIE 2018)

aprendizagem [Costa et al. 2017, Barbosa et al. 2015, Martinhago et al. 2014, Wilson e Ribas 2014, Gonçalves et al. 2013, Scaico et al. 2012]. Com relação aos cenários de aprendizagem, destaca-se a aplicação da técnica no contexto da sala de aula [Martinhago et al. 2014], laboratório de programação [Gonçalves et al. 2013], atividades culturais [Vieira et al. 2013] e de lazer [Barbosa et al. 2015], dentre outros.

Apesar da diversidade de cenários utilizados, não foram encontrados estudos na literatura que avaliassem o impacto de uso da técnica de Computação Desplugada em um cenário colaborativo (grupos de aprendizagem) fundamentado por teorias de aprendizagem colaborativa. Os grupos de aprendizagem representam a estrutura básica para a criação de cenários colaborativos em que duas ou mais pessoas devem interagir para a realização de uma tarefa. De acordo com Isotani et al. (2013), esses cenários quando fundamentados por teorias de aprendizagem colaborativa trazem benefícios a todos os participantes do grupo, uma vez que essas teorias definem condições essenciais (ex.: contexto de aprendizagem, papel dos alunos) para que os alunos aprendam de forma mais efetiva.

Dessa forma, este artigo tem como objetivo apresentar um relato de experiência sobre o uso dessa técnica em um cenário colaborativo fundamentado pela teoria de aprendizagem Peer Tutoring [Endlsey 1980].

Além dessa seção introdutória, este trabalho está organizado da seguinte forma: na Seção 2 são apresentados os trabalhos relacionados. Na sequência, a Seção 3 apresenta o método utilizado neste estudo. Na Seção 4 é apresentado o planejamento e desenvolvimento do estudo experimental. Na Seção 5 são apresentados e discutidos os resultados e, finalmente, na Seção 6 são apresentadas as conclusões.

\section{Trabalhos Relacionados}

$\mathrm{Na}$ literatura observou-se três principais frentes de pesquisa sobre o uso da técnica de computação desplugada para apoiar o ensino de computação: (1) adaptação das atividades desplugadas do livro Computer Science Unplugged [Bell et al. 2011] no intuito de atender a grupos específicos, (2) desenvolvimento de novas atividades desplugadas com o propósito de apoiar diferentes conteúdos de computação, (3) combinação da técnica de computação desplugada à outras abordagens com o objetivo de, por exemplo, dinamizar o aprendizado.

Com relação à adaptação das atividades, Scaico et al. (2012) apresentam o método de "Ensino das Lâmpadas" como uma melhoria na atividade de "Números Binários" [Bell et al. 2011] para facilitar o entendimento dos alunos do $5^{\circ}$ ao $9^{\circ}$ ano do ensino fundamental sobre o significado do " 0 " e " 1 ". O método consiste em substituir a figura do "0" por uma lâmpada apagada, e a figura do "1" por uma lâmpada acesa. Os resultados foram satisfatórios dado que os alunos conseguiram entender o conceito de número binário e desenvolver sua própria estratégia para resolução de problemas. No contexto de alunos com necessidades especiais, Wilson e Ribas (2014) relatam a experiência de uso da computação desplugada com indivíduos de 13 a 20 anos pertencentes a APAE (Associação de Pais e Amigos dos Excepcionais). No intuito de atender às necessidades desse público, foram feitas adaptações em algumas atividades do livro Computer Science Unplugged [Bell et al. 2011]. Ao final do projeto constatouse que as atividades desplugadas são aplicáveis a esse público. 
Sobre o desenvolvimento de novas atividades, Martinhago et al. (2014) propõem quatro atividades desplugadas para a disciplina de Banco de Dados para ensinar os conceitos de abstração de dados, modelo relacional e consultas SQL. Embora os resultados não tenham mostrado impacto significativo na aprendizagem dos conceitos, foi comprovada diferença positiva na satisfação dos alunos. Para a disciplina de Estrutura de Dados, Costa et al. (2017) apresentam duas atividades desplugadas para trabalhar os conteúdos de algoritmos de ordenação e tabela hash. Os resultados analisados mostraram melhora na aprendizagem dos alunos.

No que diz respeito à combinação com outras abordagens, Vieira et al. (2013) apresentam uma forma inovadora de transmitir os conceitos de computação combinando a técnica desplugada com atividades interativas no formato de peças teatrais. O projeto foi desenvolvido em escolas públicas envolvendo alunos do ensino fundamental, médio e de graduação e teve como proposta melhorar o processo de ensino e aprendizagem para professores e alunos. Barbosa et al. (2015) descrevem o desenvolvimento de uma gincana desplugada com 24 alunos do $1^{\circ}$ ano do ensino médio para trabalhar as atividades propostas no livro Computer Science Unplugged [Bell et al. 2011]. Durante as atividades foram feitas adaptações em algumas atividades do livro para que os alunos pudessem entender melhor os conceitos. Gonçalves et al. (2013) relatam a experiência de incentivar alunos do curso de Licenciatura em Computação no ensino de programação de forma lúdica como uma tentativa de tornar os conceitos de programação mais atraentes aos alunos. O trabalho consistiu no desenvolvimento de um objeto de aprendizagem desplugado (jogo EvOU) que após finalizado foi combinado a linguagem Scratch para ensinar os conectivos lógicos E, OU e NÃO, pertencentes ao conteúdo de lógica computacional. Ao final constatou-se que a experiência foi positiva ao ampliar a visão e criatividade dos alunos licenciados sobre os métodos de ensino de programação.

Apesar da relevância e contribuições dos estudos apresentados anteriormente, não foram encontrados na literatura trabalhos fundamentados por uma teoria de aprendizagem que investiguem o impacto de uso da técnica de computação desplugada em um cenário colaborativo. Logo, o presente trabalho se baseia nesse cenário para analisar os ganhos e dificuldades de aprendizagem dos alunos.

\section{Método}

O método utilizado neste trabalho baseia-se na execução de um experimento controlado quantitativo que associa a técnica da Computação Desplugada [Bell et al. 2011] à teoria de aprendizagem colaborativa Peer Tutoring [Endlsey 1980].

A Computação Desplugada, do inglês Computer Science Unplugged, é um método de ensino com o objetivo de explicar os fundamentos da computação sem a necessidade de um computador [Bell et al., 2009]. Utilizando uma linguagem de fácil entendimento, Tim Bell, Ian H. Witten e Mike Fellows escreveram o livro "Computer Science Unplugged", disponibilizado gratuitamente em vários idiomas ${ }^{1}$. O livro é composto por 12 atividades que trabalham três fundamentos da computação: representação dos dados, universo dos algoritmos e a comunicação com computadores.

\footnotetext{
${ }^{1}$ Site do projeto de Computação Desplugada: < http://csunplugged.org/>
} 
Neste artigo foi utilizada a versão traduzida para o português [Bell et al. 2011] para trabalhar o conteúdo de "Números Binários" referente ao primeiro fundamento do livro.

As teorias de aprendizagem colaborativas têm como objetivo facilitar e promover a interação dos estudantes no trabalho em grupo ao definirem algumas condições essenciais para o aprendizado. Dentre elas tem-se: o contexto de aprendizagem, o tipo de conhecimento e habilidade a serem desenvolvidas, o papel a ser desempenhado pelos estudantes e as atividades a serem desenvolvidas no grupo [Isotani et al., 2013]. Neste artigo, será utilizada a teoria de aprendizagem colaborativa Peer Tutoring [Endlsey, 1980] cujo objetivo de aprendizagem é a "Construção do Conhecimento". Com base nessa teoria, os alunos podem desempenhar um dos seguintes papéis no grupo: "tutor" (tutor) e "tutelado" (tutee). Logo, os alunos que desempenharem o papel de "tutor" devem: explicar o conteúdo, demonstrar como resolver um problema, monitorar e checar a resolução de um problema, ouvir e entender a dúvida do colega. Os alunos que desempenharem o papel de "tutelado" devem: apresentar de forma clara quais são as dúvidas, prestar atenção na explicação e/ou demonstração feita pelo colega, demonstrar se está entendendo ou não a explicação.

\section{Estudo Experimental}

\subsection{Contexto e Definição do Experimento}

A experiência relatada neste trabalho foi realizada nos meses de abril e maio de 2018 com os alunos do ensino fundamental II do Colégio $\mathrm{Anglo}^{2}$, localizado na cidade de Viçosa - MG. O colégio Anglo-Viçosa possui atualmente 1472 alunos regularmente matriculados, sendo: (i) 7 na educação especial, (ii) 72 na educação infantil, (iii) 214 no ensino fundamental I, (iv) 310 no ensino fundamental II, (v) 307 no ensino médio, e (vi) 562 na graduação e pós-graduação à distância (EAD).

Para este estudo foram selecionadas as turmas do $7^{\circ}, 8^{\circ}$ e $9^{\circ}$ anos do ensino fundamental II. O tema "Números Binários" foi escolhido por fazer parte do conteúdo programático da disciplina de Matemática das turmas do $6^{\circ}$ ano. Logo, é esperado que os alunos do $7^{\circ}, 8^{\circ}$ e $9^{\circ}$ anos já tenham tido contato com esse tópico, mesmo que superficialmente.

\subsection{Objetivo}

Este experimento teve como objetivo investigar os ganhos e dificuldades de aprendizagem dos alunos do ensino fundamental II do $7^{\circ}, 8^{\circ}$ e $9^{\circ}$ anos, ao serem inseridos em um cenário de aprendizagem colaborativo baseado na associação da técnica de computação desplugada [Bell et al. 2011] com a teoria de aprendizagem Peer Tutoring [Endlsey 1980].

\subsection{Participantes}

Para este experimento foram selecionados 162 alunos do ensino fundamental II, na faixa etária de 12 a 14 anos, pertencentes às turmas do $7^{\circ}$ ano $(\mathrm{A}$ e $\mathrm{B}), 8^{\circ}$ ano $(\mathrm{A}, \mathrm{B}$ e $\mathrm{C})$ e $9^{\circ}$ ano (A, B e C). Desse conjunto, 22 alunos estão no $7^{\circ}$ ano A, 24 alunos no $7^{\circ}$ ano B, 14

\footnotetext{
${ }^{2}$ Site do colégio Anglo-Viçosa: < http://anglovicosa2.wixsite.com/anglovicosa/o-colegio >
} 
VII Congresso Brasileiro de Informática na Educação (CBIE 2018)

Anais do XXIV Workshop de Informática na Escola (WIE 2018)

alunos no $8^{\circ}$ ano A, 28 alunos no $8^{\circ}$ ano $\mathrm{B}, 16$ alunos no $8^{\circ}$ ano $\mathrm{C}, 24$ alunos no $9^{\circ}$ ano A, 16 alunos no $9^{\circ}$ ano B e 18 alunos no $9^{\circ}$ ano C. Do total de 162 alunos, 96 são do sexo feminino e 66 do sexo masculino.

\subsection{Instrumentos}

Para este estudo foram utilizados quatro instrumentos ${ }^{3}$ detalhados a seguir.

\subsubsection{Termo de Consentimento Livre e Esclarecido}

O termo de consentimento foi elaborado pelos organizadores da pesquisa e impresso em duas vias que foram enviadas aos pais ou responsável legal de cada participante. As duas vias foram assinadas pelos responsáveis, sendo uma delas entregue aos organizadores dessa pesquisa.

\subsubsection{Testes de conhecimento}

Foram elaborados três testes de conhecimento (um para cada etapa do experimento, ver Seção 4.6) com o intuito de avaliar o domínio dos alunos sobre o conteúdo de "Números Binários" e tópicos relacionados. Cada teste permitia ao aluno atingir até 31 pontos e foi dividido em duas partes. A primeira parte avaliou o conhecimento dos alunos sobre as operações de multiplicação, potência na base 2 e padrões sequenciais. A segunda parte mediu o domínio dos alunos sobre como fazer a conversão de um número decimal (base 10) para binário (base 2) e vice e versa. Vale ressaltar que os três testes de conhecimento foram elaborados pelos organizadores da pesquisa e revisados pelos professores de Matemática do colégio Anglo-Viçosa que lecionam para as turmas do $7^{\circ}, 8^{\circ}$ e $9^{\circ}$ anos.

\subsubsection{Atividades em Grupo}

Para a dinâmica em grupo foram elaboradas três atividades, adaptadas do livro Computer Science Unplugged [Bell et al, 2011] para o tópico sobre "Números Binários". Cada atividade apresenta uma breve explicação ou revisão do conteúdo a ser trabalhado e uma parte de exercícios. Por exemplo, na "Atividade 1: Números Binários", a primeira parte explica como os cartões binários devem ser posicionados e apresenta um exemplo de como fazer a conversão de um número da base 10 (decimal) para a base 2 (binário). A intenção é que cada grupo utilize essa primeira parte para auxiliar no entendimento do conteúdo para a resolução dos exercícios da segunda parte.

Como o objetivo do trabalho em grupo é a "Construção do Conhecimento", nas atividades subsequentes, os alunos devem trabalhar em conjunto para conseguir entender o funcionamento da conversão inversa (Atividade 2), ou seja, transformar um número da base 2 (binário) para a base 10 (decimal) e transformar um número decimal acima de 31 para binário (Atividade 3 ).

\subsubsection{Cartões Binários}

Conforme sugerido pelo livro Computer Science Unplugged [Bell et al., 2011] foi elaborado um conjunto de cinco cartões de tamanho grande para demonstração do

\footnotetext{
3 Os Testes de Conhecimento e Atividades em Grupo estão disponíveis em: $<$ https://drive.google.com/drive/folders/10b15-7EDm226WS8ulXE48gCvKZwAGTcP?usp=sharing > .
} 
VII Congresso Brasileiro de Informática na Educação (CBIE 2018)

Anais do XXIV Workshop de Informática na Escola (WIE 2018)

funcionamento dos números binários. Além disso, um conjunto de cinco cartões de tamanho pequeno foi impresso para cada grupo (dupla) de alunos.

\subsection{Etapas do Experimento}

A configuração do experimento realizado neste estudo é composta por três fases: Fase A (Pré-teste), Fase B (Intervenção e Pós-teste) e Fase C (Teste de Retenção).

\subsubsection{Fase A (Pré-teste)}

No primeiro encontro, realizado na primeira semana de Abril/2018, os participantes responderam individualmente ao pré-teste de conhecimento (em papel) que teve como objetivo medir o conhecimento atual dos alunos sobre "Números Binários" e tópicos relacionados. Os alunos levaram em média 15 min para resolução do pré-teste.

\subsubsection{Fase B (Intervenção e Pós-teste)}

No segundo encontro, realizado com um intervalo de aproximadamente quinze dias da Fase A, os alunos foram divididos em dois grupos: "tutores" e "tutelados".

O grupo de "tutores" permaneceu na sala de aula com um dos pesquisadores que, utilizando os cartões binários de tamanho grande, apresentou uma breve demonstração sobre o seu uso, conforme sugerido pelo livro Computer Science Unplugged [Bell et al., 2011]. A demonstração se restringiu à conversão de um número decimal para binário. Além disso, o pesquisador também passou as orientações sobre as ações no grupo para o aluno que desempenhar o papel "tutor", por exemplo, explicar o funcionamento dos cartões, entender a dúvida do colega, dentre outros. O grupo de "tutelados" deixou a sala de aula juntamente com o professor da disciplina de Matemática que explicou as ações para os alunos que desempenharam o papel de "tutelado", por exemplo, prestar atenção na explicação do colega, fazer perguntas no caso de dúvidas, dentre outros. $\mathrm{O}$ tempo médio para a realização dessa dinâmica foi de $15 \mathrm{~min}$.

Na sequência, o grupo de "tutelados" retornou à sala de aula para que os grupos 4 fossem formados. Cada dupla recebeu um conjunto de cartões binários de tamanho pequeno para usarem nas três atividades em grupo (Seção 4.5.3). O tempo médio para realização das atividades em grupo foi de $45 \mathrm{~min}$. A Figura 1(a) e 1(b) ilustram, respectivamente, a demonstração sobre o uso dos cartões binários e uma das turmas do $8^{\circ}$ ano trabalhando em grupo.

Depois de finalizada a etapa de intervenção, os alunos responderam ao pós-teste de conhecimento (em papel) com o propósito de avaliar o quanto eles aprenderam imediatamente após o desenvolvimento das atividades em grupo. O tempo médio para realização do pós-teste de conhecimento foi de $35 \mathrm{~min}$.

\subsubsection{Fase C (Teste de retenção)}

No terceiro encontro, realizado com um intervalo de aproximadamente quinze dias da Fase B, foi aplicado o teste de retenção de conhecimento (em papel). Este teste teve

\footnotetext{
${ }^{4}$ As duplas de trabalho, bem como o papel que os alunos irão desempenhar no grupo, foram definidos a partir da execução do algoritmo de formação de grupos desenvolvido por Isotani et al. (2010).
} 
VII Congresso Brasileiro de Informática na Educação (CBIE 2018)

Anais do XXIV Workshop de Informática na Escola (WIE 2018)

como propósito avaliar o conhecimento retido pelos alunos após um período. O tempo médio para realização do teste de retenção foi de $30 \mathrm{~min}$.

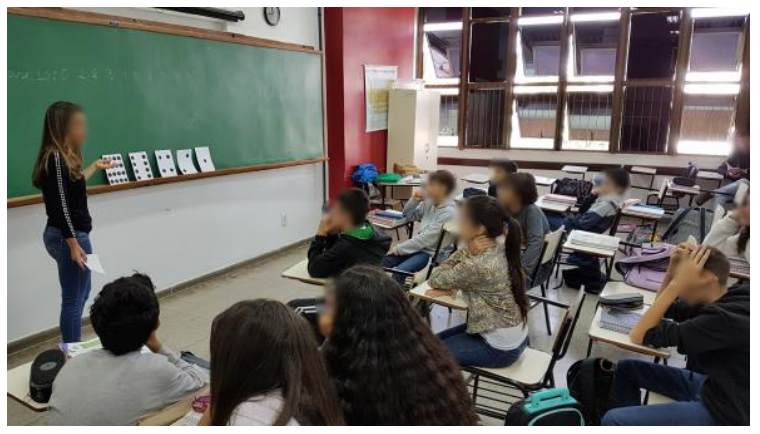

(a)

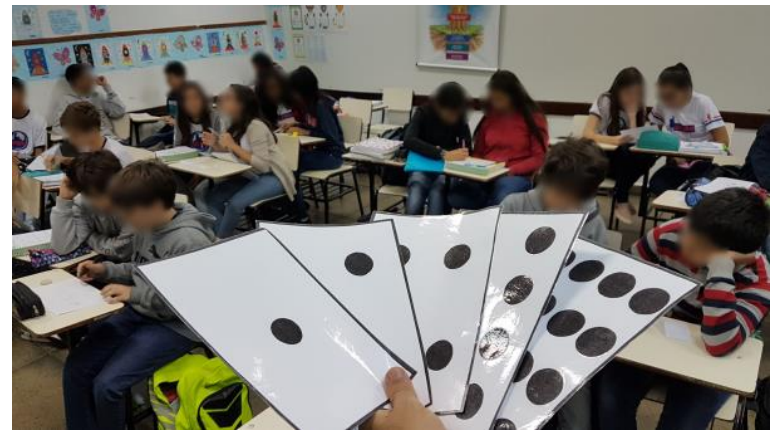

(b)

Figura 1. Foto ilustrando: (a) a demonstração de como utilizar os cartões binários e (b) uma das turmas do 8ㅇ ano trabalhando em grupo (duplas).

\section{Resultados e Discussão}

O problema investigado neste estudo é norteado pela seguinte questão de pesquisa: “Os alunos tiveram ganho de aprendizagem significativo ao serem inseridos em um cenário de aprendizagem colaborativo baseado na associação da técnica de computação desplugada à teoria de aprendizagem Peer Tutoring?".

Para responder à questão de pesquisa, foram analisados os ganhos de aprendizagem obtidos pelos 162 alunos em duas situações ${ }^{5}$ : (1) imediatamente após a intervenção (nota do pós-teste), e (2) após o período de 15 dias da intervenção (nota do teste de retenção).

Na situação (1), a média de acertos no pós-teste de conhecimento $(\mu=24,43)$ foi significativamente maior $(p<, 001)$ do que a média de acertos no pré-teste $(\mu=12,61)$. $\mathrm{Na}$ situação (2), a média de acertos no teste de retenção $(\mu=21,40)$ foi também significativamente maior $(p<, 001)$ do que a média de acertos no pré-teste. Essa análise mostra que tanto o aprendizado quanto a retenção de conhecimento foram efetivos, pois, os alunos acertaram significativamente mais questões imediatamente após a intervenção (pós-teste) e após o período de aproximadamente 15 dias da intervenção (teste de retenção). Considerando que a análise anterior teve significância estatística, é possível responder à questão de pesquisa afirmando que: "sim, os alunos tiveram ganho significativo de aprendizagem ao serem inseridos no cenário colaborativo proposto".

A Figura 1 apresenta o número de alunos que tiveram ganho positivo no pósteste e teste de retenção, distribuídos por faixa de pontos, ou seja, um aluno que tirou 11 pontos no pré-teste e 29 pontos no pós-teste teve um ganho de 18 pontos, logo ele se encontra na faixa de 16-20 pontos. Com base nessa figura pode-se observar também que a quantidade de alunos na faixa de 6 a 10 pontos e 11 a 15 pontos no pós-teste e teste de retenção é bem similar. Além disso, percebe-se uma concentração maior de alunos com ganho de aprendizagem na faixa de 16 a 20 pontos no pós-teste (70) e teste de retenção

\footnotetext{
${ }^{5}$ Para análise estatística dos dados foi utilizado o teste- $t$.
} 
VII Congresso Brasileiro de Informática na Educação (CBIE 2018)

Anais do XXIV Workshop de Informática na Escola (WIE 2018)

(44), o que é positivo dado que esses alunos elevaram consideravelmente suas notas após a intervenção, atingindo em alguns casos o total de pontos do teste (31 pontos).

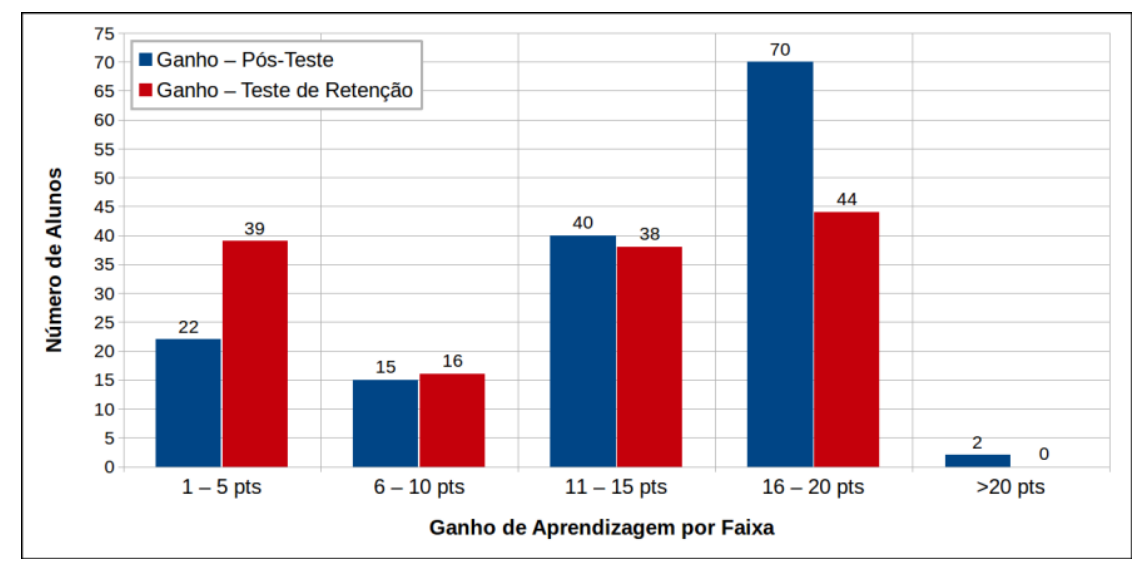

Figure 2. Número de alunos que tiveram ganho de aprendizagem positivo no pós-teste (149 alunos) e teste de retenção (137 alunos) distribuídos por faixa de pontos.

Com relação às dificuldades dos alunos, a Tabela 1 apresenta um resumo dos principais erros cometidos pelos alunos nos testes de conhecimento durante o processo de conversão decimal-binário e binário-decimal após o uso dos cartões binários. Como os cartões binários não haviam sido introduzidos aos alunos na Fase A do experimento, o resultado do pré-teste de conhecimento não será considerado nesta análise.

Tabela 1. Percentual dos principais erros nos testes de conhecimento.

\begin{tabular}{|c|l|c|c|c|}
\cline { 2 - 4 } \multicolumn{1}{c|}{} & \multicolumn{1}{c|}{ Tipo de Erro } & $\begin{array}{c}\text { (Atividade 1) } \\
\text { Conversão } \\
\text { Decimal } \rightarrow \text { Binário }\end{array}$ & $\begin{array}{c}\text { (Atividade 2) } \\
\text { Conversão } \\
\text { Binário } \rightarrow \text { Decimal }\end{array}$ & $\begin{array}{c}\text { (Atividade 3) } \\
\text { Conversão } \\
\text { Decimal > 31 } \rightarrow \text { Binário }\end{array}$ \\
\hline $\begin{array}{c}\text { Fase B } \\
\text { Pós-teste }\end{array}$ & $\begin{array}{c}\text { Cálculo ou falta } \\
\text { de entendimento }\end{array}$ & $\begin{array}{c}28,66 \% \\
(45 \text { alunos })\end{array}$ & $\begin{array}{c}48,73 \% \\
(77 \text { alunos })\end{array}$ & $\begin{array}{c}25,17 \% \\
(38 \text { alunos })\end{array}$ \\
\cline { 2 - 5 } & $\begin{array}{c}\text { Inversão dos } \\
\text { Cartões Binários }\end{array}$ & $\begin{array}{c}10,19 \% \\
(16 \text { alunos })\end{array}$ & $\begin{array}{c}9,49 \% \\
(15 \text { alunos })\end{array}$ & $\begin{array}{c}9,27 \% \\
(14 \text { alunos })\end{array}$ \\
\hline \multirow{2}{*}{$\begin{array}{c}\text { Fase C } \\
\text { Retenção }\end{array}$} & $\begin{array}{l}\text { Cálculo ou falta } \\
\text { de entendimento }\end{array}$ & $\begin{array}{c}27,21 \% \\
(40 \text { alunos })\end{array}$ & $\begin{array}{c}42,18 \% \\
(62 \text { alunos })\end{array}$ & $\begin{array}{c}30,50 \% \\
(43 \text { alunos })\end{array}$ \\
\cline { 2 - 5 } & Inversão dos & $\begin{array}{c}21,77 \% \\
(32 \text { alunos })\end{array}$ & $\begin{array}{c}21,77 \% \\
(32 \text { alunos })\end{array}$ \\
\hline
\end{tabular}

Com base na Tabela 1, os principais tipos de erros cometidos pelos alunos no processo de conversão decimal-binário, binário-decimal ocorreram devido a: (1) erros de cálculo ou falta de entendimento do conteúdo, (2) inversão dos cartões binários ${ }^{6}$.

Em ambos os testes de conhecimento (pós-teste e retenção) pode-se observar que os erros por cálculo ou falta de entendimento do conteúdo ocorreram em maior percentual do que os erros por inversão dos cartões binários. Se analisarmos isoladamente cada um dos tipos de erro, pode-se notar que o percentual de alunos que

\footnotetext{
${ }^{6} \mathrm{O}$ erro de "inversão dos cartões binários" consiste no posicionamento incorreto dos cartões, ou seja, ao invés dos alunos posicionarem os cartões na ordem $16-8$ - 4 - 2 - 1, os cartões foram colocados na ordem inversa: $1-2-4-8-16$.
} 
VII Congresso Brasileiro de Informática na Educação (CBIE 2018)

Anais do XXIV Workshop de Informática na Escola (WIE 2018)

cometeram o erro de cálculo ou falta de entendimento no pós-teste é muito próximo do percentual de alunos que cometeram o erro no teste de retenção. Por outro lado, o percentual de alunos que cometeram o erro de inversão dos cartões dobrou do pós-teste para o teste de retenção, em todos os três casos de conversão. Uma justificativa para este aumento deve-se ao fato dos cartões binários, usados para demonstração, terem fícado expostos na lousa durante toda a Fase B do experimento, ou seja, durante as atividades em grupo e na realização do pós-teste de conhecimento.

Um fato curioso relacionado à inversão dos cartões é que, apesar do aumento do número de alunos que cometeram esse tipo de erro do pós-teste para o teste de retenção, quatro alunos que fizeram a inversão no pós-teste não fizeram no teste de retenção. Além disso, esses alunos aumentaram sua nota do primeiro (pós-teste) para o segundo (retenção) teste, ou seja, todos ficaram com nota acima de 25 pontos no teste de retenção.

Com relação aos tipos de conversão, verificou-se em ambos os testes (pós-teste e retenção) que os alunos tiveram maior dificuldade na conversão binário-decimal do que nos outros casos. Essa dificuldade pode ter sido ocasionada pelo fato da demonstração realizada ao aluno "tutor" na Fase B ter sido restrita à conversão decimal-binário.

\section{Conclusão}

Este trabalho apresentou um relato de experiência sobre o uso da técnica de Computação Desplugada [Bell et al. 2011] em um cenário colaborativo fundamentado pela teoria de aprendizagem Peer Tutoring [Endlsey 1980]. Essa investigação foi realizada por meio de um estudo experimental com 162 alunos do $7^{\circ}, 8^{\circ}$ e $9^{\circ}$ anos do ensino fundamental II. Os resultados mostraram uma melhora significativa tanto no aprendizado imediato dos alunos (logo após a intervenção) quanto no aprendizado retido ( $\sim 15$ dias após a intervenção).

Dentre os principais tipos de erros cometidos pelos alunos destaca-se (1) os erros de cálculo ou falta de entendimento do conteúdo e (2) os erros por inversão dos cartões binários. Apesar dos erros por inversão dos cartões terem ocorrido em menor número, os autores acreditam que ele poderia ter sido evitado ou minimizado, caso o posicionamento dos cartões tivesse sido enfatizado na demonstração feita pelo pesquisador para os alunos que iriam desempenhar o papel de "tutor". Observou-se também que os alunos tiveram maior dificuldade na conversão binário-decimal do que na conversão decimal-binário.

Com base nos resultados obtidos observou-se que o aprendizado por meio do uso da técnica de computação desplugada em um cenário colaborativo fundamentado por uma teoria de aprendizagem foi significativo. Além disso, os alunos tiveram a oportunidade de trabalhar em grupo na construção do conhecimento sobre conversão de números binários para decimal (e vice e versa) sem o auxílio do professor e pesquisadores. Essa iniciativa vai de encontro com o construtivismo que apresenta o indivíduo como o sujeito ativo no processo de aprendizagem, que por meio da prática colaborativa possibilita sua reflexão e o desenvolvimento de novas soluções [Pozo, 2002]. 
VII Congresso Brasileiro de Informática na Educação (CBIE 2018)

Anais do XXIV Workshop de Informática na Escola (WIE 2018)

Como trabalho futuro sugere-se a investigação do uso da técnica de computação desplugada em um cenário colaborativo fundamentado por teorias de aprendizagem para outras atividades do livro Computer Science Unplugged [Bell et al., 2011].

\section{Agradecimentos}

Os autores agradecem ao CNPq, Capes e FAPESP pelo apoio financeiro e a colaboração do Colégio Anglo-Viçosa, em especial, à diretora Lúcia Duque, coordenadora Emiliane e professores Danilo, Fernanda e Janaíne.

\section{Referências}

Bell, T., Alexander, J., Freeman, I., Grimley, M. (2009). Computer Science Unplugged: School students doing real computing without computers. The New Zealand Journal of Applied Computing and Information Technology, 13(1):20-29.

Bell, T., Witten, I., Fellows, M. (2011). "Computer Science Unplugged - Ensinando Ciência da Computação sem o uso do Computador". Tradução de Luciano Porto Barreto. Disponível em: http://csunplugged.org/. Acesso em 15/06/2018.

Barbosa, A. V. de S., Neto, A. F. P., de Oliveira, R. N. R., da Costa, T. L. S., Souto, A. L., de Araújo, O., Costa, F. V. S. (2015). "O ensino de conceitos computacionais para alunos do ensino médio: relato de experiência de uma gincana e das estratégias utilizadas pelos alunos na resolução das atividades desplugadas, XXI Workshop sobre Educação em Computação (CSBC).

Costa, T. L. S. da, Souza, F. V. C., Costa, W. E. (2017). "O uso de Computação Desplugada para apoiar a Aprendizagem de Algoritmos de Ordenação e Tabela Hash". Trabalho de Conclusão de Curso, Universidade Federal da Paraíba.

Endlsey, W. R. (1980). Peer tutorial instruction. Englewood Cliffs, NJ: Educational Technology.

Gonçalves, D. A. S., Silva, G. M. da, Luz, R. S. da, Silva, C. E. P. (2013). "Relato de experiência de alunos do curso de Licenciatura em Computação do IFMG - campus Ouro Branco na utilização de objetos de aprendizagem desplugados e do Scratch como instrumentos no ensino de programação", II Congresso Brasileiro de Informática na Educação (CBIE 2013), p. 335-344.

Isotani, S., Inaba, A., Ikeda, M., Mizoguchi, R. (2009). An ontology engineering approach to the realization of theory-driven group formation. International Journal of Computer-Supported Collaborative Learning, 4(4): 445-478.

Isotani, S., Mizoguchi, R., Isotani, S., Capeli, O. M., Isotani, N., Albuquerque, A. R. P. L., Bittencourt, I. I., Jaques, P. (2013). A Semantic Web-based authoring tool to facilitate the planning of collaborative learning scenarios compliant with learning theories. Computers and Education, 63: 267-284.

Martinhago, A. Z., Smarzaro, R., Lima, I. Guimarães, L. (2014). "Computação Desplugada no Ensino de Bancos de Dados na Educação Superior", XXII Workshop sobre Educação em Computação (WEI- CSBC), p. 1307-1315.

Pozo, J. I. (2002). Aprendizes e mestres. Porto Alegre: Artmed.

Scaico, P., Henrique, M., Cunha, F., Alencar, Y. (2012). Um Relato de Experiências de Estagiários da Licenciatura em Computação com o Ensino de Computação para Crianças. Revista Renote: Novas Tecnologias na Educação, 10(3).

Vieira, A., Passos, O., Barreto, R. (2013). "Um Relato de Experiência do Uso da Técnica Computação Desplugada", XXXIII Congresso da Sociedade Brasileira de Computação (CSBC), p. 671-680.

Wilson, R. E., Ribas, S. G. (2014). "Estudo da aplicabilidade do projeto unplugged com crianças especiais", Encontro Anual de Tecnologia da Informação e Semana Acadêmica de Tecnologia da Informação, $n^{\circ}$ 1, p. 247-254. 